\title{
REMARKS ON THE LIFESPAN \\ OF THE SOLUTIONS TO SOME MODELS OF INCOMPRESSIBLE FLUID MECHANICS
}

\author{
RAPHAËL DANCHIN \\ (Communicated by Walter Craig)
}

\begin{abstract}
We give lower bounds for the lifespan of a solution to the inviscid Boussinesq system. In dimension two, we point out that it tends to infinity when the initial (relative) temperature tends to zero. This is, to the best of our knowledge, the first result of this kind for the inviscid Boussinesq system. In passing, we provide continuation criteria (of independent interest) in the $N$-dimensional case. In the second part of the paper, our method is adapted to handle the axisymmetric incompressible Euler equations with swirl.
\end{abstract}

\section{INTRODUCTION}

The evolution of the velocity $u=u(t, x)$ and pressure $P=P(t, x)$ fields of a perfect homogeneous incompressible fluid is governed by the following Euler equations:

$$
\left\{\begin{array}{l}
\partial_{t} u+u \cdot \nabla u+\nabla P=0, \\
\operatorname{div} u=0
\end{array}\right.
$$

There is a huge amount of literature concerning the well-posedness issue for Euler equations. Roughly, they may be solved locally in time in any reasonable Banach space embedded in the set $C^{0,1}$ of bounded Lipschitz functions (see e.g. [1, 4, 6, 12, 13, 17, 19, 22]).

In the two-dimensional case, it is well known that Euler equations are globally well-posed for sufficiently smooth initial data. This noticeable fact relies on the conservation of the vorticity $\omega:=\partial_{1} u^{2}-\partial_{2} u^{1}$ along the flow of the velocity field and has been first proved rigorously in the pioneering works by W. Wolibner [20] and V. Yudovich [21].

This conservation property is no longer true, however, in more physically relevant contexts such as

(1) the three-dimensional setting for (0.1),

(2) nonhomogeneous incompressible perfect fluids,

(3) inviscid fluids subjected to a buoyancy force which is advected by the velocity fluid (the so-called inviscid Boussinesq system below).

As a consequence, the problem of global existence for general (even smooth or small) data is still open for the above three cases.

Received by the editors September 15, 2011.

2010 Mathematics Subject Classification. Primary 35Q35, 76B03, 76B70, 35A01.

(C) 2012 American Mathematical Society Reverts to public domain 28 years from publication 
In recent work [9, it has been shown that for slightly nonhomogeneous twodimensional incompressible fluids, the lifespan tends to infinity when the nonhomogeneity tends to zero. The present paper is mainly dedicated to the study of the lifespan for the first and third items.

More precisely, in the first section of the paper, we shall consider the inviscid Boussinesq system:

$$
\left\{\begin{array}{l}
\partial_{t} \theta+u \cdot \nabla \theta=0, \\
\partial_{t} u+u \cdot \nabla u+\nabla P=\theta e_{N}, \\
\operatorname{div} u=0 .
\end{array}\right.
$$

Here the relative temperature $\theta=\theta(t, x)$ is a real-valued function 11 and $e_{N}$ stands for the unit vertical vector.

As for the standard incompressible Euler equations, any functional space embedded in $C^{0,1}$ is a good candidate for the study of the well-posedness issue for (0.2). This stems from the fact that System (0.2) is a coupling between transport equations. Hence preserving the initial regularity requires the velocity field to be at least locally Lipschitz with respect to the space variable. By arguing as in [1, Chap. 7], one may show that, indeed, System (0.2) is locally well-posed in $B_{p, q}^{s}$ whenever $B_{p, q}^{s}$ is embedded in $C^{0,1}$ or, in other words, for any $(s, p, q) \in \mathbb{R} \times[1,+\infty]^{2}$ satisfying

$$
s>1+\frac{N}{p} \quad \text { or } \quad s=1+\frac{N}{p} \text { and } q=1 .
$$

As a by-product of estimates for transport equations, we shall get various continuation criteria which generalize those of [1] and of [16]. We shall finally establish lower bounds for the lifespan of the solutions to (0.2) which show that in the two-dimensional case and for small initial temperature, the solution tends to be global-in-time.

As pointed out in many works (see e.g. 11]), there is a formal similarity between the two-dimensional Boussinesq system and general axisymmetric solutions to the three-dimensional Euler system - the so-called axisymmetric solutions with swirl. In the second part of this paper, we adapt the method of the first part so as to establish new lower bounds for the lifespan to those solutions in the case where the swirl is small. In particular, we find out that the solution tends to be global if the swirl goes to zero.

In the Appendix, we briefly recall the definition and a few basic properties of Besov space and prove a commutator estimate.

Before going further into the description of our results, let us introduce some notation.

- Throughout the paper, $C$ stands for a harmless "constant", the meaning of which depends on the context.

- The vorticity $\omega$ associated to a vector field $u$ over $\mathbb{R}^{N}$ is the matrix-valued function with entries

$$
\omega_{i j}:=\partial_{j} u^{i}-\partial_{i} u^{j} .
$$

If $N=2$, then the vorticity is identified with the scalar function $\omega:=$ $\partial_{1} u^{2}-\partial_{2} u^{1}$ and if $N=3$, with the vector field $\nabla \times u$.

\footnotetext{
${ }^{1}$ It need not be nonnegative as it designates the discrepancy to some reference temperature.
} 
- For every Banach space $X$ and interval $I$ of $\mathbb{R}$, we denote by $\mathcal{C}(I ; X)$ the set of continuous functions on $I$ with values in $X$. If $X$ has predual $X^{*}$, then we denote by $\mathcal{C}_{w}(I ; X)$ the set of bounded measurable functions $f: I \rightarrow X$ such that for any $\phi \in X^{*}$, the function $t \mapsto\langle f(t), \phi\rangle_{X \times X^{*}}$ is continuous over $I$.

\section{The InVISCID BoussinesQ SYSTEM}

This section is devoted to the well-posedness issue for the inviscid Boussinesq system (0.2). We first establish a local-in-time existence result and continuation criteria in the spirit of those for the incompressible Euler equation. Next, we provide a new lower bound for the lifespan. Roughly, we establish that if $\theta_{0}$ is of order $\varepsilon$, then the lifespan is at least of order $\log |\log \varepsilon|$.

1.1. Well-posedness and continuation criteria. The present subsection is devoted to the proof of the following result.

Theorem 1.1. Let $(s, p, q) \in \mathbb{R} \times[1,+\infty]^{2}$ satisfy (0.3). Assume that $u_{0}$ (with $\left.\operatorname{div} u_{0} \equiv 0\right)$ and $\theta_{0}$ belong to $B_{p, q}^{s}$ and that, in addition, $\left(u_{0}, \nabla \theta_{0}\right) \in L^{r}$ for some $r \in] 1, \infty[$ if $p=\infty$. Then (0.2) admits a unique local-in-time solution $(\theta, u, \nabla P)$ in $\mathcal{C}_{w}(]-T, T\left[; B_{p, \infty}^{s}\right)$ if $q=\infty$ and in $\mathcal{C}(]-T, T\left[; B_{p, q}^{s}\right)$ if $q<\infty$. Besides, $\nabla \theta$ and $u$ are in $\mathcal{C}(]-T, T\left[; L^{r}\right)$ if $\left(u_{0}, \nabla \theta_{0}\right) \in L^{r}$.

Furthermore, the solution may be continued beyond $\square^{2} T$ whenever one of the following three conditions is satisfied:
i) $\int_{0}^{T}\|\nabla u\|_{L^{\infty}} d t<\infty$
ii) $\int_{0}^{T}\left(\|\omega\|_{L^{\infty}}+\|\nabla \theta\|_{L^{\infty}}\right) d t<\infty$ and $s>1+N / p$;
iii) $N=2, \int_{0}^{T}\|\nabla \theta\|_{L^{\infty}} d t<\infty$ and $s>1+2 / p$.

Before proving this result, a few comments are in order.

1. If it is assumed that $\omega_{0} \in L^{r}$ instead of $u_{0} \in L^{r}$, then the vorticity of the constructed solution is continuous in time with values in $L^{r}$.

2. In the two-dimensional case and in the Hölder spaces framework, the above statement has been established in [7]. The critical Besov case (that is, $p=1+2 / p, p \in] 1, \infty[$ ) has been investigated in [16].

3. In [11, a continuation criterion involving the $L^{\infty}$ norm of the vorticity only has been stated. However, as the first inequality after (7) in [11 fails if $m \geq 2$, we do not know whether that criterion is correct.

4. The first item has been proved recently in [16] in the two-dimensional case.

5 . Let us finally mention that one may replace $\|\omega\|_{L^{\infty}}$ with $\|\omega\|_{\dot{B}_{\infty, \infty}^{0} \cap L^{r}}$ in the second criterion.

Proof of Theorem 1.1. The proof of the local well-posedness in the Besov spaces framework is a straightforward adaptation to that of the corresponding result for the Euler system in $B_{p, q}^{s}$, and is thus omitted. The reader may refer to [1, Chap. 7] for more details.

\footnotetext{
${ }^{2}$ For expository purposes, we just consider positive times.
} 
So let us go for the proof of the continuation criteria. Let us first assume that $1<$ $p<\infty$. In this case, the Marcinkiewicz theorem for Calderón-Zygmund operators ensures that

$$
\|\nabla u\|_{L^{p}} \leq C\|\omega\|_{L^{p}} .
$$

Therefore, decomposing $\omega$ into low and high frequencies as follows $3^{3}$

$$
\omega=\Delta_{-1} \omega+\left(\operatorname{Id}-\Delta_{-1}\right) \omega,
$$

and taking advantage of the remark that follows Proposition A.1 in the appendix, we gather that

$$
\|\nabla u\|_{B_{p, q}^{s-1}} \leq C\|\omega\|_{B_{p, q}^{s-1}}
$$

Now, in dimension $N$ the vorticity equation reads $\partial_{t} \omega+u \cdot \nabla \omega+\mathcal{A}(\nabla u, \omega)={ }^{T} \nabla\left(\theta e_{N}\right)-\nabla\left(\theta e_{N}\right) \quad$ with $\mathcal{A}(\nabla u, \omega):=\omega \cdot \nabla u+{ }^{T} \nabla u \cdot \omega$. Hence applying $\Delta_{j}$ to the vorticity equation yields

$$
\partial_{t} \omega_{j}+u \cdot \nabla \omega_{j}=-\Delta_{j} \mathcal{A}(\nabla u, \omega)+\Delta_{j}\left({ }^{T} \nabla\left(\theta e_{N}\right)-\nabla\left(\theta e_{N}\right)\right)+\left[u, \Delta_{j}\right] \cdot \nabla \omega
$$

with $\omega_{j}:=\Delta_{j} \omega$ and $\theta_{j}:=\Delta_{j} \theta$. Therefore, because $\operatorname{div} u=0$,

$$
\begin{aligned}
\left\|\omega_{j}(t)\right\|_{L^{p}} \leq\left\|\omega_{j}(0)\right\|_{L^{p}} & +\int_{0}^{t}\left\|\nabla \theta_{j}\right\|_{L^{p}} d \tau \\
& +\int_{0}^{t}\left\|\Delta_{j} \mathcal{A}(\nabla u, \omega)\right\|_{L^{p}} d \tau+\int_{0}^{t}\left\|\left[u, \Delta_{j}\right] \cdot \nabla \omega\right\|_{L^{p}} d \tau .
\end{aligned}
$$

Next, let us use (see the appendix) that

$$
\left\|2^{j(s-1)}\right\|\left[u, \Delta_{j}\right] \cdot \nabla \omega\left\|_{L^{p}}\right\|_{\ell^{q}} \lesssim\|\nabla u\|_{L^{\infty}}\|\omega\|_{B_{p, q}^{s-1}} \quad \text { whenever } s>0 .
$$

If $s>1+N / p$, then standard tame estimates (see e.g. [1, Chap. 2) imply that

$$
\begin{aligned}
\|\mathcal{A}(\nabla u, \omega)\|_{B_{p, q}^{s-1}} & \leq C\left(\|\omega\|_{L^{\infty}}\|\nabla u\|_{B_{p, q}^{s-1}}+\|\nabla u\|_{L^{\infty}}\|\omega\|_{B_{p, q}^{s-1}}\right) \\
& \leq C\|\nabla u\|_{L^{\infty}}\|\nabla u\|_{B_{p, q}^{s-1}} .
\end{aligned}
$$

The last inequality remains true in the limit case $s=1+N / p$ and $q=1$, a consequence of the algebraic structure of $\mathcal{A}(\nabla u, \omega)$ (see e.g. Inequality (52) in [9]).

Hence, multiplying (1.3) by $2^{j(s-1)}$, taking the $\ell^{q}$ norm with respect to $j$ and taking advantage of (1.2) yields

$$
\|\omega(t)\|_{B_{p, q}^{s-1}} \leq\left\|\omega_{0}\right\|_{B_{p, q}^{s-1}}+C \int_{0}^{t}\|\nabla \theta\|_{B_{p, q}^{s-1}} d \tau+C \int_{0}^{t}\|\nabla u\|_{L^{\infty}}\|\omega\|_{B_{p, q}^{s-1}} d \tau .
$$

Next, in order to bound the $B_{p, q}^{s}$ norm of $\theta$, we use the fact that

$$
\partial_{t} \theta_{j}+u \cdot \nabla \theta_{j}=\left[u, \Delta_{j}\right] \cdot \nabla \theta,
$$

whence

$$
\left\|\theta_{j}(t)\right\|_{L^{p}} \leq\left\|\theta_{j}(0)\right\|_{L^{p}}+\int_{0}^{t}\left\|\left[u, \Delta_{j}\right] \cdot \nabla \theta\right\|_{L^{p}} d \tau .
$$

Given that, according to (a slight modification of) Lemma 2.100 of [1], we have

$$
\left\|2^{j s}\right\|\left[u, \Delta_{j}\right] \cdot \nabla \theta\left\|_{L^{p}}\right\|_{\ell^{q}} \lesssim\|\nabla u\|_{L^{\infty}}\|\theta\|_{B_{p, q}^{s}}+\|\nabla \theta\|_{L^{\infty}}\|\omega\|_{B_{p, q}^{s-1}},
$$

\footnotetext{
${ }^{3}$ The notation $\Delta_{-1}$ is defined in the appendix.
} 
we eventually get

$$
\|\theta(t)\|_{B_{p, q}^{s}} \leq\left\|\theta_{0}\right\|_{B_{p, q}^{s}}+C \int_{0}^{t}\left(\|\nabla u\|_{L^{\infty}}\|\theta\|_{B_{p, q}^{s}}+\|\nabla \theta\|_{L^{\infty}}\|\omega\|_{B_{p, q}^{s-1}}\right) d \tau .
$$

Finally, from the equation for $\theta$, we easily get

$$
\|\nabla \theta(t)\|_{L^{\infty}} \leq\left\|\nabla \theta_{0}\right\|_{L^{\infty}}+\int_{0}^{t}\|\nabla u\|_{L^{\infty}}\|\nabla \theta\|_{L^{\infty}} d \tau .
$$

So if $\nabla u$ is in $L^{1}\left(\left[0, T\left[; L^{\infty}\right)\right.\right.$, then $\nabla \theta$ is in $L^{\infty}\left(\left[0, T\left[\times \mathbb{R}^{N}\right)\right.\right.$. Therefore, summing up Inequalities (1.5) and (1.8) and using Gronwall's lemma, we easily deduce that $\|\omega\|_{B_{p, r}^{s-1}}$ and $\|\theta\|_{B_{p, r}^{s}}$ are bounded on $[0, T[$. To complete the proof of the boundedness of the solution in $L^{\infty}\left(\left[0, T\left[; B_{p, r}^{s}\right)\right.\right.$, we still have to bound $u$ in $L^{\infty}\left(\left[0, T\left[; L^{p}\right)\right.\right.$. For that, we use the fact that

$$
u(t)=u(0)-\int_{0}^{t} \mathcal{P}(u \cdot \nabla u) d \tau
$$

where $\mathcal{P}$ stands for the Leray projector over divergence-free vector fields. As it is continuous over $L^{p}$ (recall that $1<p<\infty$ ), we deduce that

$$
\|u(t)\|_{L^{p}} \leq\left\|u_{0}\right\|_{L^{p}}+C \int_{0}^{t}\|\nabla u\|_{L^{\infty}}\|u\|_{L^{p}} d \tau .
$$

Now, the standard continuation criterion for hyperbolic PDEs ensures that the solution $(\theta, u)$ may be continued beyond $T$.

Let us now treat the case where $s>1+N / p$ and

$$
\int_{0}^{T}\left(\|\omega\|_{L^{\infty}}+\|\nabla \theta\|_{L^{\infty}}\right) d t<\infty .
$$

We first bound $\omega$ and $\nabla \theta$ in $L^{\infty}\left(\left[0, T\left[; L^{p}\right)\right.\right.$ by taking advantage of (1.1) and of the vorticity and temperature equations. We get

$$
\begin{aligned}
& \|\omega(t)\|_{L^{p}} \leq\left\|\omega_{0}\right\|_{L^{p}}+\int_{0}^{t}\|\nabla \theta\|_{L^{p}} d \tau+C \int_{0}^{t}\|\omega\|_{L^{p}}\|\omega\|_{L^{\infty}} d \tau, \\
& \|\nabla \theta(t)\|_{L^{p}} \leq\left\|\nabla \theta_{0}\right\|_{L^{p}}+C \int_{0}^{t}\|\nabla \theta\|_{L^{\infty}}\|\omega\|_{L^{p}} d \tau .
\end{aligned}
$$

Hence,

$$
\|(\omega, \nabla \theta)(t)\|_{L^{p}} \leq\left\|\left(\omega_{0}, \nabla \theta_{0}\right)\right\|_{L^{p}}+C \int_{0}^{t}\left(1+\|(\omega, \nabla \theta)\|_{L^{\infty}}\right)\|(\omega, \nabla \theta)\|_{L^{p}} d \tau .
$$

So Gronwall's lemma provides us with a bound for $\omega$ and $\nabla \theta$ in $L^{\infty}\left(\left[0, T\left[; L^{p}\right)\right.\right.$.

Next, we use the following classical logarithmic interpolation inequality (see e.g. [1]):

$$
\|\nabla u\|_{L^{\infty}} \lesssim\|\omega\|_{L^{p} \cap L^{\infty}} \log \left(e+\|\omega\|_{B_{p, q}^{s-1}}\right) .
$$

Plugging this inequality into (1.5) and (1.8) and summing, we get

$$
\begin{aligned}
& \|\omega(t)\|_{B_{p, q}^{s-1}}+\|\theta(t)\|_{B_{p, q}^{s}} \leq\left\|\omega_{0}\right\|_{B_{p, q}^{s-1}}+\left\|\theta_{0}\right\|_{B_{p, q}^{s}} \\
& \quad+C \int_{0}^{t}\left(1+\|\nabla \theta\|_{L^{\infty}}+\|\omega\|_{L^{p} \cap L^{\infty}}\right)\left(\|\omega\|_{B_{p, q}^{s-1}}+\|\theta\|_{B_{p, q}^{s}}\right) \log \left(e+\|\omega\|_{B_{p, q}^{s-1}}\right) d \tau .
\end{aligned}
$$


So Osgood's lemma implies that $\|\omega\|_{B_{p, r}^{s-1}}$ and $\|\theta\|_{B_{p, r}^{s}}$ are bounded on $[0, T[$. Bounding $\|u\|_{L^{p}}$ may be done by combining Inequalities (1.11) and (1.15). Hence the solution $(\theta, u)$ may be continued beyond $T$.

Let us finally assume that $N=2$ and that

$$
\int_{0}^{T}\|\nabla \theta\|_{L^{\infty}} d t<\infty .
$$

Then Equation (1.16) gives

$$
\|\omega(t)\|_{L^{\infty}} \leq\left\|\omega_{0}\right\|_{L^{\infty}}+\int_{0}^{t}\left\|\partial_{1} \theta\right\|_{L^{\infty}} d \tau .
$$

Hence $\omega \in L^{\infty}\left(\left[0, T\left[\times \mathbb{R}^{2}\right)\right.\right.$ and the previous continuation criterion implies that the solution $(\theta, u)$ may be continued beyond $T$.

Let us end the proof with a few comments concerning the cases $p=1, \infty$. If $p=\infty$ and the solution also satisfies $(\nabla \theta, \omega) \in L^{\infty}\left(\left[0, T\left[; L^{r}\right)\right.\right.$ for some $\left.r \in\right] 1, \infty[$, then arguing as for proving (1.2) yields

$$
\|\nabla u\|_{B_{\infty, q}^{s-1} \cap L^{r}} \leq C\|\omega\|_{B_{\infty, q}^{s-1} \cap L^{r}} .
$$

From the vorticity and temperature equations, we get

$$
\begin{aligned}
\|\omega(t)\|_{L^{r}} & \leq\left\|\omega_{0}\right\|_{L^{r}}+2 \int_{0}^{t}\|\nabla u\|_{L^{\infty}}\|\omega\|_{L^{r}} d \tau+2 \int_{0}^{t}\|\nabla \theta\|_{L^{r}} d \tau, \\
\|\nabla \theta(t)\|_{L^{r}} & \leq\left\|\nabla \theta_{0}\right\|_{L^{r}}+\int_{0}^{t}\|\nabla u\|_{L^{\infty}}\|\nabla \theta\|_{L^{r}} d \tau .
\end{aligned}
$$

So one may conclude that (1.5) and (1.8) hold true if the norm in $B_{\infty, q}^{s-1}$ is replaced by the norm in $B_{\infty, q}^{s-1} \cap L^{r}$. In order to bound $\|u\|_{B_{\infty, q}^{s}}$, one may write that (using Bernstein's inequality to get the second line),

$$
\begin{aligned}
\|u(t)\|_{B_{\infty, q}^{s}} & \lesssim\left\|\Delta_{-1} u(t)\right\|_{L^{\infty}}+\|\omega(t)\|_{B_{\infty, q}^{s-1}} \\
& \lesssim\left\|\Delta_{-1} u_{0}\right\|_{L^{\infty}}+\left\|\Delta_{-1}\left(u(t)-u_{0}\right)\right\|_{L^{r}}+\|\omega(t)\|_{B_{\infty, q}^{s-1}} .
\end{aligned}
$$

Now, according to (1.10), we have

$$
\left\|u(t)-u_{0}\right\|_{L^{r}} \leq C \int_{0}^{t}\|u\|_{L^{r}}\|\nabla u\|_{L^{\infty}} d \tau .
$$

From this, it is easy to complete the proof.

Finally, if $p=1$, then embedding ensures that $\nabla \theta$ and $u$ are in $L^{\infty}\left(\left[0, T\left[; L^{r}\right)\right.\right.$ for some finite $r$, so that one may conclude as in the case $p=\infty$.

1.2. Lower bounds for the lifespan of the solutions to (0.2). Let $\left(\theta_{0}, u_{0}\right)$ satisfy the assumptions of Theorem 1.1. Then it is clear that $\left(\theta^{\varepsilon}, u^{\varepsilon}, \nabla \Pi^{\varepsilon}\right)$ satisfies (0.2) on $\left[T^{-} / \varepsilon, T^{+} / \varepsilon\right]$ with initial data

$$
\theta_{0}^{\varepsilon}=\varepsilon^{2} \theta_{0} \quad \text { and } \quad u_{0}^{\varepsilon}=\varepsilon u_{0}
$$

if and only if the triplet $(\theta, u, \nabla \Pi)$ defined by

$$
\theta^{\varepsilon}(t, x):=\varepsilon^{2} \theta(\varepsilon t, x), \quad u^{\varepsilon}(t, x):=\varepsilon u(\varepsilon t, x) \text { and } \Pi^{\varepsilon}(t, x):=\varepsilon^{2} \Pi(\varepsilon t, x)
$$

satisfies 0.2 on $\left[T^{-}, T^{+}\right]$with data $\left(\theta_{0}, u_{0}\right)$.

From this, we gather that for initial temperature and velocity of size $\varepsilon^{2}$ and $\varepsilon$, respectively, the lifespan is (at least) of order $\varepsilon^{-1}$. 
The above result is, obviously, independent of the dimension. At the same time, in the case $\theta_{0} \equiv 0$ (corresponding to the incompressible Euler equation) global existence holds true in dimension 2 . In the case $\theta_{0} \not \equiv 0$, the question of global existence has remained unsolved, even in the two-dimensional case. We here want to study whether, nevertheless, dimension 2 is somehow "better". To answer this question, we shall take advantage of the fact that the vorticity equation in dimension 2 has no stretching term: it reduces to

$$
\partial_{t} \omega+u \cdot \nabla \omega=\partial_{1} \theta
$$

Hence, taking advantage of the special a priori estimates for the transport equation in Besov spaces with null regularity index (as discovered by M. Vishik in [19] and by T. Hmidi and S. Keraani in [13]), one may write

$$
\|\omega(t)\|_{B_{\infty, 1}^{0}} \leq\left(\left\|\omega_{0}\right\|_{B_{\infty, 1}^{0}}+\int_{0}^{t}\left\|\partial_{1} \theta\right\|_{B_{\infty, 1}^{0}} d \tau\right)\left(1+C \int_{0}^{t}\|\nabla u\|_{L^{\infty}} d \tau\right) .
$$

This will be the key to our result below.

Theorem 1.2. Assume that $N=2$. Let $\left(\theta_{0}, u_{0}\right)$ be in $B_{p, q}^{s}$ with $(s, p, q)$ satisfying (0.3). If $p \in\{1,+\infty\}$, suppose in addition that $\left(\nabla \theta_{0}, \omega_{0}\right) \in L^{r}$ for some $1<r<\infty$. There exists a constant $C$ depending only on $r$ and such that (setting $p=r$ if $p \in(1,+\infty))$ the lifespan $T^{*}$ of (0.2) satisfies

$$
T^{*} \geq \frac{1}{C\left\|\omega_{0}\right\|_{B_{\infty, 1}^{0} \cap L^{r}}} \log \left(1+\frac{1}{2} \log \left(1+\frac{C\left\|\omega_{0}\right\|_{B_{\infty, 1}^{0} \cap L^{r}}^{2}}{\left\|\nabla \theta_{0}\right\|_{B_{\infty, 1}^{0} \cap L^{r}}}\right)\right) .
$$

Proof. Let us first notice that, according to the continuation criteria derived in Theorem 1.1] it suffices to show that if the solution is defined on $\left[0, T\left[\times \mathbb{R}^{n}\right.\right.$ with $T \leq T^{*}$ and $T^{*}$ as above, then $\omega$ and $\nabla \theta$ are bounded in $L^{\infty}\left(0, T ; B_{\infty, 1}^{0} \cap L^{r}\right)$.

Now, estimates for the transport equation in Besov spaces (see e.g. [1, Chap. 3]) yield

$$
\|\nabla \theta(t)\|_{B_{\infty, 1}^{0}} \leq\left\|\nabla \theta_{0}\right\|_{B_{\infty, 1}^{0}} e^{C \int_{0}^{t}\|\nabla u\|_{B_{\infty, 1}^{0}} d \tau}
$$

Of course, standard $L^{r}$ estimates for the transport equation imply that (1.19)

$$
\|\omega(t)\|_{L^{r}} \leq\left\|\omega_{0}\right\|_{L^{r}}+\int_{0}^{t}\left\|\partial_{1} \theta\right\|_{L^{r}} \quad \text { and } \quad\|\nabla \theta(t)\|_{L^{r}} \leq\left\|\nabla \theta_{0}\right\|_{L^{r}} e^{\int_{0}^{t}\|\nabla u\|_{L^{\infty} d \tau}} .
$$

Let us finally notice that putting together embedding, Inequality (1.1) and the remark that follows Proposition A.1, we have

$$
\|\nabla u\|_{L^{\infty}} \lesssim\|\nabla u\|_{B_{\infty, 1}^{0}} \lesssim\|\omega\|_{B_{\infty, 1}^{0} \cap L^{r}}
$$

Therefore, denoting

$$
\Omega(t):=\|\omega(t)\|_{B_{\infty, 1}^{0} \cap L^{r}} \quad \text { and } \quad \Theta(t):=\|\nabla \theta(t)\|_{B_{\infty, 1}^{0} \cap L^{r}}
$$

and taking advantage of (1.17), (1.18), (1.19), we conclude that

$$
\begin{aligned}
& \Theta(t) \leq \Theta_{0} e^{C \int_{0}^{t} \Omega d \tau}, \\
& \Omega(t) \leq\left(\Omega_{0}+\int_{0}^{t} \Theta d \tau\right)\left(1+C \int_{0}^{t} \Omega d \tau\right) .
\end{aligned}
$$


Now, plugging the inequality for $\Theta(t)$ into the inequality for $\Omega(t)$, we get

$$
\Omega(t) \leq\left(\Omega_{0}+t \Theta_{0} e^{C \int_{0}^{t} \Omega d \tau}\right)\left(1+C \int_{0}^{t} \Omega d \tau\right) .
$$

Let us assume for a while that

$$
T \Theta_{0} e^{C \int_{0}^{T} \Omega d \tau} \leq \Omega_{0} .
$$

Then (1.20) and Gronwall's lemma imply that

$$
\Omega(t) \leq 2 \Omega_{0} e^{2 C t \Omega_{0}} \quad \text { for all } t \in[0, T] .
$$

Therefore, for Condition (1.21) to be satisfied, it suffices that

$$
\Theta_{0} T \exp \left(e^{2 C T \Omega_{0}}-1\right) \leq \Omega_{0}
$$

that is to say,

$$
X \exp \left(e^{X}-1\right) \leq Y \quad \text { with } \quad X:=2 C T \Omega_{0} \quad \text { and } \quad Y=\frac{2 C \Omega_{0}^{2}}{\Theta_{0}} .
$$

Let us notice that

$$
X \leq e^{X}-1 \leq \exp \left(e^{X}-1\right)-1 \quad \text { for any } X \in \mathbb{R}^{+} .
$$

Hence Inequality (1.23) is satisfied provided that

$$
\exp \left(2\left(e^{X}-1\right)\right) \leq 1+Y \text {. }
$$

So we easily gather from a bootstrap argument that the lifespan $T^{*}$ satisfies

$$
T^{*} \geq \frac{1}{2 C \Omega_{0}} \log \left(1+\frac{1}{2} \log \left(1+\frac{2 C \Omega_{0}^{2}}{\Theta_{0}}\right)\right),
$$

which is exactly the desired inequality.

Remark 1.3. In the case where the solution is $C^{1, r}$ for some $r \in(0,1)$ (an assumption which is not satisfied in the critical regularity case), one may first write estimates for $\|\omega\|_{L^{\infty}}$ and $\|\omega\|_{C^{r}}$, and next use the classical logarithmic inequality for bounding $\|\nabla u\|_{L^{\infty}}$ in terms of $\|\omega\|_{L^{\infty}}$ and $\|\omega\|_{C^{r}}$. This does not improve the lower bound for the lifespan, though.

\section{The AXISYmmetric InCOMPREssible Euler EQuations}

We now consider the incompressible Euler equations (0.1). As recalled in the introduction, Euler equations are globally well-posed in dimension 2. In dimension $d \geq 3$, the global well-posedness issue has remained unsolved unless some property of symmetry is satisfied: it is known that axisymmetric or helicoidal without swirl data generate global solutions (see e.g. [8] and the references therein for more details).

In the general case, an easy scaling argument similar to that of the Boussinesq system yields that for data of size $\varepsilon$, the lifespan is at least of order $\varepsilon^{-1}$.

Here we want to focus on the axisymmetric solutions to Euler equations with swirl, that is, on solutions $u$ to (0.1) such that, in cylindrical coordinates,

$$
u(r, z)=u^{r}(r, z) e_{r}+u^{\theta}(r, z) e_{\theta}+u^{z}(r, z) e_{z} .
$$


Recall that the corresponding vorticity reads $\omega(r, z)=\omega^{r}(r, z) e_{r}+\omega^{\theta}(r, z) e_{\theta}+$ $\omega^{z}(r, z) e_{z}$ with

$$
\omega^{r}(r, z)=-\partial_{z} u^{\theta} e_{r}, \quad \omega^{\theta}(r, z)=\partial_{z} u^{r}-\partial_{r} u^{z}, \quad \omega^{z}(r, z)=\frac{1}{r} \partial_{r}\left(r u^{\theta}\right) .
$$

With this notation, axisymmetric solutions satisfy (see e.g. 4])

$$
\left\{\begin{array}{l}
\widetilde{D}_{t} u^{r}+\partial_{r} \Pi=r^{-1}\left(u^{\theta}\right)^{2}, \\
\widetilde{D}_{t} u^{\theta}=-r^{-1} u^{r} u^{\theta}, \\
\widetilde{D}_{t} u^{z}+\partial_{z} \Pi=0, \\
\partial_{r}\left(r u^{r}\right)+\partial_{z}\left(r u^{z}\right)=0,
\end{array} \quad \text { with } \widetilde{D}_{t}:=\partial_{t}+u^{r} \partial_{r}+u^{z} \partial_{z} .\right.
$$

As pointed out in [11, there is a striking similarity between the two-dimensional Boussinesq system (0.2) satisfied by $(\theta, \omega)$ in the previous section, and the equations satisfied by $\left(u^{\theta}, \omega^{\theta}\right)$ here. Indeed,

$$
\left\{\begin{array}{l}
\widetilde{D}_{t}\left(r u^{\theta}\right)=0, \\
\widetilde{D}_{t} \omega^{\theta}-\frac{1}{r} u^{r} \omega^{\theta}-\frac{1}{r} \partial_{z}\left(u^{\theta}\right)^{2}=0
\end{array}\right.
$$

whence, denoting $\Gamma:=\left(r u^{\theta}\right)^{2}$ and $\zeta:=r^{-1} \omega^{\theta}$, we have

$$
\widetilde{D}_{t} \Gamma=0 \quad \text { and } \quad \widetilde{D}_{t} \zeta=\frac{1}{r^{4}} \partial_{z} \Gamma .
$$

Therefore, up to the singular coefficient $1 / r^{4}$, the functions $\Gamma=\Gamma(r, z)$ and $\zeta=$ $\zeta(r, z)$ play the same role as the temperature and the vorticity, respectively, in the $2 \mathrm{D}$ Boussinesq system. Keeping in mind that data such that $u_{0}^{\theta} \equiv 0$ generate global solutions, it is natural to study whether having $r^{-1} \omega_{0}^{\theta}=\mathcal{O}(1)$ and $r u_{0}^{\theta}=\mathcal{O}(\varepsilon)$ gives rise to a family of solutions with lifespan going to infinity when $\varepsilon$ goes to 0 .

For technical reasons, however, due to the singularity near the axis, we shall consider the axisymmetric Euler equations in a smooth bounded axisymmetric domain $\Omega$ of $\mathbb{R}^{3}$ such that, for some given $0<r_{0}<R_{0}$,

$$
\Omega \subset\left\{(x, y, z) \in \mathbb{R}^{3}: r_{0}<\sqrt{x^{2}+y^{2}}<R_{0}\right\} .
$$

Let us first give a local well-posedness result for the Euler equation in a domain:

Theorem 2.1. Let $(s, p, q)$ satisfy Condition (0.3). Let $u_{0}$ be in $B_{p, q}^{s}(\Omega)$ with $\operatorname{div} u_{0}=0$ and $u_{0}$ tangent to the boundary of $\Omega$. Then System (0.1) with slip boundary conditions has a unique local solution $u$ in $\mathcal{C}_{w}(]-T, T\left[; B_{p, q}^{s}(\Omega)\right.$ ) (or in $\mathcal{C}(]-T, T\left[; B_{p, q}^{s}(\Omega)\right)$ if $\left.q<\infty\right)$.

If in addition $u_{0}$ satisfies (2.1), then $u$ satisfies (2.2).

Proof. This statement has been essentially proved by A. Dutrifoy in 10 except in the critical case $s=1+3 / p$ and $r=1$. However, the critical case may be handled by the same method 4 as it relies on a priori estimates for transport equations which are also true in this case.

The last part of the statement is a classical consequence of the uniqueness and of the symmetry of the data $u_{0}$.

One can now state the main result of this part.

\footnotetext{
${ }^{4}$ Proving a continuation criterion involving the vorticity was the main purpose of Dutrifoy's paper, and this requires that $s>1+3 / p$. This is probably the reason why the statement in the critical case is not given therein.
} 
Theorem 2.2. Let $u_{0}$ be an axisymmetric divergence-free vector field in $B_{p, q}^{s}(\Omega)$ with $(s, p, q)$ satisfying (0.3) and $\Omega$ a bounded domain satisfying (2.4). Suppose in addition that $\left.u_{0}\right|_{\partial \Omega}$ is tangent to the boundary of $\Omega$. Then the lifespan $T^{*}$ to the solution of (0.1) satisfies

$$
T^{*} \geq \frac{1}{C\left\|\omega_{0}^{\theta}\right\|_{B_{\infty, 1}^{0}}} \log \left(1+\frac{1}{2} \log \left(1+\frac{C\left\|\omega_{0}^{\theta}\right\|_{B_{\infty, 1}^{0}}}{\left\|\left(u_{0}^{\theta}\right)^{2}\right\|_{B_{\infty, 1}^{1}}}\right)\right)
$$

for some constant $C$ depending only on $\Omega$.

Proof. It suffices to bound the norm of $u$ in $B_{\infty, 1}^{1}$ as it controls high norms (see [10] and notice that $B_{\infty, 1}^{1}$ embeds in $\left.C^{0,1}\right)$. Let $\widetilde{u}:=u^{r} e_{r}+u^{z} e_{z}$. Denote by $\widetilde{\psi}$ the solution given by Proposition A.4 to the elliptic equation

$$
\left\{\begin{array}{ccc}
-\Delta \widetilde{\psi}=\omega^{\theta} e_{\theta} & \text { in } \quad & \Omega, \\
\partial_{n} \widetilde{\psi}=0 & \text { on } & \partial \Omega,
\end{array} \quad \int_{\Omega} \widetilde{\psi} d x=0 .\right.
$$

Notice that

$$
\operatorname{div} \widetilde{u}=0=\operatorname{div}(\nabla \wedge \widetilde{\psi}) \text { and that } \nabla \wedge \widetilde{u}=\omega^{\theta} e_{\theta}=\nabla \wedge(\nabla \wedge \widetilde{\psi}) .
$$

As, in addition, both $\widetilde{u}$ and $\nabla \wedge \widetilde{\psi}$ have null circulation on the components of $\partial \Omega$ (a consequence of the symmetry properties of those two functions and of the domain), they coincide. Hence, Proposition A.4 ensures that

$$
\|\nabla \widetilde{u}\|_{B_{\infty, 1}^{0}} \leq C\left\|\omega^{\theta} e_{\theta}\right\|_{B_{\infty, 1}^{0}} .
$$

This inequality will enable us to adapt to the axisymmetric Euler equations the proof of lower bounds for the lifespan of solutions.

We proceed as follows. According to the work by A. Dutrifoy (see in particular Prop. 6 and Cor. 5 in [10]) for the transport equation in a smooth bounded domain, estimates in Besov spaces $B_{p, q}^{s}(\Omega)$ are the same as in the whole space case. From this, one may deduce by following the method of 13 that in the particular case $s=0$, the estimates improve (as in (1.17)). So we get, bearing (2.3) in mind:

$$
\|\zeta(t)\|_{B_{\infty, 1}^{0}} \leq\left(\left\|\zeta_{0}\right\|_{B_{\infty, 1}^{0}}+\int_{0}^{t}\left\|r^{-4} \partial_{z} \Gamma\right\|_{B_{\infty, 1}^{0}} d \tau\right)\left(1+C \int_{0}^{t}\|\nabla \widetilde{u}\|_{L^{\infty}} d \tau\right) .
$$

General Dutrifoy's estimates for the transport equation also imply that

$$
\|\Gamma(t)\|_{B_{\infty, 1}^{1}} \leq\left\|\Gamma_{0}\right\|_{B_{\infty, 1}^{1}} \exp \left(C \int_{0}^{t}\|\nabla \widetilde{u}\|_{B_{\infty, 1}^{0}} d \tau\right) .
$$

Now, the important observation is that $1 / r^{4}$ is in $C^{0,1}(\Omega)$ (for $r \geq r_{0}$ in $\Omega$ ). Hence

$$
\left\|r^{-4} \partial_{z} \Gamma\right\|_{B_{\infty, 1}^{0}} \leq C\left\|\partial_{z} \Gamma\right\|_{B_{\infty, 1}^{0}}
$$

whence

$$
\|\zeta(t)\|_{B_{\infty, 1}^{0}} \leq\left(\left\|\zeta_{0}\right\|_{B_{\infty, 1}^{0}}+C \int_{0}^{t}\left\|\partial_{z} \Gamma\right\|_{B_{\infty, 1}^{0}} d \tau\right)\left(1+C \int_{0}^{t}\|\nabla \widetilde{u}\|_{L^{\infty}} d \tau\right) .
$$

Finally, according to (2.5) and classical embedding properties, we have

$$
\|\nabla \widetilde{u}\|_{L^{\infty}} \lesssim\|\nabla \widetilde{u}\|_{B_{\infty, 1}^{0}} \lesssim\left\|\omega^{\theta} e_{\theta}\right\|_{B_{\infty, 1}^{0}} .
$$

As $\omega^{\theta} e_{\theta}=\zeta r e_{\theta}$ and, under our assumption on $\Omega, r e_{\theta}$ is in $C^{0,1}$, one may thus conclude that

$$
\|\nabla \widetilde{u}\|_{L^{\infty}} \lesssim\|\zeta\|_{B_{\infty, 1}^{0}}
$$


From this point, one may proceed exactly as for the Boussinesq system; we deduce the following lower bound for the lifespan of the solution:

$$
T^{*} \geq \frac{1}{C\left\|\zeta_{0}\right\|_{B_{\infty, 1}^{0}}} \log \left(1+\frac{1}{2} \log \left(1+\frac{C\left\|\zeta_{0}\right\|_{B_{\infty, 1}^{0}}}{\left\|\Gamma_{0}^{2}\right\|_{B_{\infty, 1}^{1}}}\right)\right)
$$

Of course, owing to the shape of $\Omega$, up to an irrelevant constant, one may replace $\zeta_{0}$ with $\omega_{0}^{\theta}$ and $r u_{0}^{\theta}$ with $u_{0}^{\theta}$, respectively.

Remark 2.3. We believe Theorem 2.2 to be true in the case where $\Omega$ satisfying (2.4) is unbounded. However, we refrained from giving the statement as we did not find in the literature the counterpart of Theorem 2.1 and of Proposition A.4.

Let us emphasize however that unbounded domains have been considered in 2 . (Hölder spaces) and [14, 15] (weighted Sobolev spaces). By following Dutrifoy's approach, we do not see any obstruction to obtaining similar results in the Besov space framework. This is only a matter of having suitable extension operators available for the domain considered.

We also believe that Proposition A.4 may be extended to unbounded domains provided we prescribe some condition at infinity: the inequality

$$
\|\nabla \widetilde{u}\|_{B_{\infty, 1}^{0} \cap L^{r}} \leq C\left\|\omega^{\theta} e_{\theta}\right\|_{B_{\infty, 1}^{0} \cap L^{r}}
$$

for any $r \in] 1,+\infty[$ seems to be reasonable. However, as proving such inequalities is not the point of this paper, we restricted ourselves to bounded domains.

\section{Appendix A}

In this appendix, we recall the definition and a few properties of nonhomogeneous Besov spaces $B_{p, q}^{s}$, then prove a commutator estimate.

Let us first introduce a dyadic partition of unity with respect to the Fourier variable (the so-called Littlewood-Paley decomposition): we fix a smooth radial function $\chi$ supported in (say) the ball $B(0,4 / 3)$, equal to 1 in a neighborhood of $B(0,3 / 4)$ and such that $r \mapsto \chi\left(r e_{r}\right)$ is nonincreasing over $\mathbb{R}_{+}$, and set $\varphi(\xi)=$ $\chi(\xi / 2)-\chi(\xi)$.

The dyadic blocks $\left(\Delta_{j}\right)_{j \in \mathbb{Z}}$ are defined by

$$
\Delta_{j}:=0 \text { if } j \leq-2, \quad \Delta_{-1}:=\chi(D) \quad \text { and } \quad \Delta_{j}:=\varphi\left(2^{-j} D\right) \text { if } j \geq 0 .
$$

It may be easily checked that the identity $u=\sum_{j} \Delta_{j} u$ holds true in the sense of tempered distributions.

One can now define the Besov space $B_{p, q}^{s}$ as the set of tempered distributions $u$ so that $\|u\|_{B_{p, q}^{s}}$ is finite, where

$$
\|u\|_{B_{p, q}^{s}}:=\left(\sum_{j} 2^{q j s}\left\|\Delta_{j} u\right\|_{L^{p}}^{q}\right)^{\frac{1}{q}} \text { if } q<\infty \quad \text { and } \quad\|u\|_{B_{p, \infty}^{s}}:=\sup _{j}\left(2^{j s}\left\|\Delta_{j} u\right\|_{L^{p}}\right) .
$$

Roughly speaking, the elements of $B_{p, q}^{s}$ have " $s$ derivatives in $L^{p}$ ". For instance, the Besov space $B_{2,2}^{s}$ coincides with the nonhomogeneous Sobolev space $H^{s}$ (for any $s \in \mathbb{R}$ ), and $B_{\infty, \infty}^{s}$ coincides with the Hölder space $C^{s}$, if $s \in \mathbb{R}_{+} \backslash \mathbb{N}$. 
In this paper, we use freely the following properties for Besov spaces (see e.g. 1, Chap. 2]):

Proposition A.1. Let $(s, p, q) \in \mathbb{R} \times[1,+\infty]^{2}$.

- The Besov space $B_{p, q}^{s}$ is (continuously) embedded in the set $C^{0,1}$ of Lipschitz bounded functions if and only if Condition (0.3) is satisfied.

- The gradient operator maps $B_{p, q}^{s}$ in $B_{p, q}^{s-1}$.

- More generally, if $F: \mathbb{R}^{N} \rightarrow \mathbb{R}$ is a smooth homogeneous function of degree $m$ away from a neighborhood of the origin, then for all $(p, q) \in[1, \infty]^{2}$ and $s \in \mathbb{R}$, operator $F(D)$ maps $B_{p, q}^{s}$ in $B_{p, q}^{s-m}$.

Remark A.2. From the last property, given that the Biot-Savart operator $\mathcal{B}: \omega \mapsto$ $\nabla u$ is a homogeneous smooth multiplier of degree 0 , we deduce that $\left(\operatorname{Id}-\Delta_{-1}\right) \mathcal{B}$ is a self-map on $B_{p, q}^{s}$ for any $s \in \mathbb{R}$ and $1 \leq p, q \leq \infty$. This implies Inequality (1.2).

The definition of Besov spaces may be extended by restriction to general domains $\Omega$ of $\mathbb{R}^{N}$ :

Definition A.3. Let $\Omega$ be a domain of $\mathbb{R}^{N}$, and $(s, p, q) \in \mathbb{R} \times[1,+\infty]^{2}$. We denote by $B_{p, q}^{s}(\Omega)$ the set of distributions $u$ over $\Omega$ which are the restriction (in the sense of distributions) to some $\widetilde{u}$ in $B_{p, q}^{s}\left(\mathbb{R}^{N}\right)$. The space $B_{p, q}^{s}(\Omega)$ is endowed with the norm

$$
\|u\|_{B_{p, q}^{s}(\Omega)}:=\inf \|\widetilde{u}\|_{B_{p, q}^{s}\left(\mathbb{R}^{N}\right)},
$$

where the infimum is taken over the set of $\widetilde{u}$ in $B_{p, q}^{s}\left(\mathbb{R}^{N}\right)$ such that $u$ coincides with the restriction of $\widetilde{u}$ to $\Omega$.

The following result will be needed in the proof of Inequality (2.5).

Proposition A.4. Let $\Omega$ be a smooth bounded domain of $\mathbb{R}^{N}$ and $\omega$ be in $B_{\infty, 1}^{0}(\Omega)$. If in addition the mean value of $\omega$ on $\Omega$ is zero, then the Neumann equation

$$
\left\{\begin{array}{lll}
-\Delta \psi=\omega & \text { in } & \Omega, \\
\partial_{n} \psi=0 & \text { on } & \partial \Omega,
\end{array} \quad \int_{\Omega} \psi d x=0,\right.
$$

has a unique solution $\psi$ in $B_{\infty, 1}^{2}(\Omega)$ and we have

$$
\left\|\nabla^{2} \psi\right\|_{B_{\infty, 1}^{0}(\Omega)} \leq C\|\omega\|_{B_{\infty, 1}^{0}(\Omega)} .
$$

Proof. In [18, Th. 4.4], it has been proved that, for any $s>-1$, if $\omega \in C^{s}(\Omega):=$ $B_{\infty, \infty}^{s}(\Omega)$ (with 0 mean value), then the above system has a unique solution $\psi$ in $C^{s+2}(\Omega)$ satisfying

$$
\left\|\nabla^{2} \psi\right\|_{C^{s}(\Omega)} \leq C\|\omega\|_{C^{s}(\Omega)} .
$$

So denoting $T: \omega \mapsto \nabla^{2} \psi$, the result follows by interpolation : it is only a matter of using the fact that $B_{\infty, 1}^{0}(\Omega)=\left(C^{-1 / 2}(\Omega), C^{1 / 2}(\Omega)\right)_{1 / 2,1}$.

Let us now turn to the proof of Inequality (1.4). Let $\widetilde{u}:=u-\Delta_{-1} u$. We decompose the commutator as follows:

$$
\left[u, \Delta_{j}\right] \cdot \nabla \omega=\sum_{i=1}^{6} R_{j}^{i}
$$


with, using the summation convention over repeated indices,

$$
\begin{aligned}
R_{j}^{1}:=\left[T_{\widetilde{u}^{k}}, \Delta_{j}\right] \partial_{k} \omega, & R_{j}^{2}:=T_{\partial_{k} \Delta_{j} \omega} \widetilde{u}^{k}, \\
R_{j}^{3}:=-\Delta_{j} T_{\partial_{k} \omega} \widetilde{u}^{k}, & R_{j}^{4}:=\partial_{k} R\left(\widetilde{u}^{k}, \Delta_{j} \omega\right), \\
R_{j}^{5}:=-\partial_{k} \Delta_{j} R\left(\widetilde{u}^{k}, \omega\right), & R_{j}^{6}:=\left[\Delta_{-1} \widetilde{u}^{k}, \Delta_{j}\right] \partial_{k} \omega .
\end{aligned}
$$

Above, $T$ and $R$ stand for the paraproduct and remainder operators, respectively, which are defined as follows (after J.-M. Bony in [5]):

$T_{f} g:=\sum_{j} S_{j-1} f \Delta_{j} g$ and $R(f, g):=\sum_{j} \sum_{\left|j^{\prime}-j\right| \leq 1} \Delta_{j} f \Delta_{j^{\prime}} g \quad$ with $\quad S_{j}:=\sum_{j^{\prime}<j} \Delta_{j}$.

Decomposition (A.1) is obtained after noticing that

$$
f g=T_{f} g+T_{g} f+R(f, g) .
$$

Let us now go to the proof of Inequality (1.4). In all that follows, $\left(c_{j}\right)_{j \geq-1}$ stands for a sequence such that $\left\|\left(c_{j}\right)\right\|_{\ell^{q}}=1$.

From [1, Lemma 2.99], we get, for $i \in\{1,6\}$,

$$
\left\|R_{j}^{i}\right\|_{L^{p}} \lesssim c_{j} 2^{-j(s-1)}\|\nabla u\|_{L^{\infty}}\|\omega\|_{B_{p, q}^{s-1}}
$$

As regards $R_{j}^{2}$, we write that

$$
R_{j}^{2}=\sum_{j^{\prime} \geq j-1} S_{j^{\prime}-1} \partial_{k} \Delta_{j} \omega \Delta_{j^{\prime}} \widetilde{u}^{k}
$$

Note that $\mathcal{F}\left(\Delta_{j} \omega\right)$ is supported in an annulus of size $2^{j}$. Hence Bernstein's Inequality ensures that

whence

$$
\left\|R_{j}^{2}\right\|_{L^{p}} \lesssim 2^{j} \sum_{j^{\prime} \geq j-1}\|\omega\|_{L^{\infty}}\left\|\Delta_{j^{\prime}} \widetilde{u}\right\|_{L^{p}}
$$

$$
\left\|R_{j}^{2}\right\|_{L^{p}} \lesssim 2^{-j(s-1)}\|\omega\|_{L^{\infty}} \sum_{j^{\prime} \geq j-1} 2^{\left(j-j^{\prime}\right) s} 2^{j^{\prime} s}\left\|\Delta_{j^{\prime}} \widetilde{u}\right\|_{L^{p}},
$$

so that we get if $s>0$,

$$
\left\|R_{j}^{2}\right\|_{L^{p}} \lesssim c_{j} 2^{-j(s-1)}\|\omega\|_{L^{\infty}}\|\widetilde{u}\|_{B_{p, q}^{s}}
$$

As for $R_{j}^{3}$, standard continuity results for the paraproduct operator (see e.g. [1, Chap. 2]) imply that

$$
\left\|R_{j}^{3}\right\|_{L^{p}} \lesssim c_{j} 2^{-j(s-1)}\|\omega\|_{L^{\infty}}\|\widetilde{u}\|_{B_{p, q}^{s}} .
$$

For $R_{j}^{4}$, one may write that

$$
R_{j}^{4}=\partial_{k} \sum_{\left|j^{\prime}-j\right| \leq 2} \Delta_{j^{\prime}} \widetilde{u}^{k} \Delta_{j}\left(\Delta_{j^{\prime}-1}+\Delta_{j^{\prime}}+\Delta_{j^{\prime}+1}\right) \omega .
$$

Hence, in view of Bernstein's inequality,

$$
\left\|R_{j}^{4}\right\|_{L^{p}} \lesssim 2^{j} \sum_{\left|j^{\prime}-j\right| \leq 2}\|\omega\|_{L^{\infty}}\left\|\Delta_{j^{\prime}} \widetilde{u}\right\|_{L^{p}} .
$$

So we get

$$
\left\|R_{j}^{4}\right\|_{L^{p}} \lesssim c_{j} 2^{-j(s-1)}\|\omega\|_{L^{\infty}}\|\widetilde{u}\|_{B_{p, q}^{s}} .
$$

Next, standard continuity results for the remainder operator yield if $s>0$,

$$
\left\|\partial_{k} R\left(\widetilde{u}^{k}, \omega\right)\right\|_{B_{p, q}^{s-1}} \lesssim\|\omega\|_{L^{\infty}}\|\widetilde{u}\|_{B_{p, q}^{s}} .
$$


Hence

$$
\left\|R_{j}^{5}\right\|_{L^{p}} \lesssim c_{j} 2^{-j(s-1)}\|\omega\|_{L^{\infty}}\|\widetilde{u}\|_{B_{p, q}^{s}} .
$$

Finally, let us notice that the operator $\omega \mapsto\left(\operatorname{Id}-\Delta_{-1}\right) u$ satisfies the hypothesis of the last item of Proposition A.1 with $m=-1$; hence

$$
\|\widetilde{u}\|_{B_{p, q}^{s}} \lesssim\|\omega\|_{B_{p, q}^{s-1}} .
$$

So putting all the above inequalities together completes the proof of (1.4).

\section{ACKNOWLEDGMENTS}

The author is indebted to O. Glass and F. Sueur for pointing out references 14, 15, 18.

\section{REFERENCES}

1. H. Bahouri, J.-Y. Chemin and R. Danchin: Fourier Analysis and Nonlinear Partial Differential Equations, Grundlehren der mathematischen Wissenschaften, 343, Springer (2011). MR2768550(2011m:35004)

2. C. Bardos and U. Frisch: Finite-time regularity for bounded and unbounded ideal incompressible fluids using Hölder estimates. Turbulence and Navier-Stokes equations, Lecture Notes in Math., 565, Springer, Berlin, pages 1-13 (1976). MR0467034 (57:6903)

3. J. Beale, T. Kato and A. Majda: Remarks on the breakdown of smooth solutions for the 3-D Euler equations, Communications in Mathematical Physics, 94(1), pages 61-66 (1984). MR763762 (85j:35154)

4. A. Bertozzi and A. Majda: Vorticity and incompressible flow, 27, Cambridge Texts in Applied Mathematics, Cambridge University Press (2002). MR,1867882 (2003a:76002)

5. J.-M. Bony: Calcul symbolique et propagation des singularités pour les équations aux dérivées partielles non linéaires, Ann. Sci. École Norm. Sup., 14(4), pages 209-246 (1981). MR631751 (84h:35177)

6. J.-Y. Chemin: Fluides parfaits incompressibles, Astérisque, 230 (1995). MR.1340046 (97d:76007)

7. D. Chae, S.-K. Kim and H.-S. Nam: Local existence and blow-up criterion of Hölder continuous solutions of the Boussinesq equations, Nagoya Mathematical Journal, 155, pages 55-80 (1999). MR:1711383 (2000g:35172)

8. R. Danchin: Axisymmetric incompressible flows with bounded vorticity, Russian Mathematical Surveys, 62(3), pages 73-94 (2007). MR2355419(2009a:76044)

9. R. Danchin and F. Fanelli: The well-posedness issue for the density-dependent Euler equations in endpoint Besov spaces, Journal de Mathématiques Pures et Appliquées, 96, pages 253-278 (2011). MR2831582

10. A. Dutrifoy: Precise regularity results for the Euler equations, Journal of Mathematical Analysis and Applications, 282(1), pages 177-200 (2003). MR.2000337 (2004i:35254)

11. W. E and C.-W. Shu: Small-scale structures in Boussinesq convection, Physics of Fluids, 6(1) pages 49-58 (1994). MR1252833(94i:76075)

12. T. Hmidi and S. Keraani: Existence globale pour le système d'Euler incompressible 2-D dans $B_{\infty, 1}^{1}$, Compte-rendu de l'Académie des Sciences, Paris, Série I, 341(11), pages 655-658 (2005). MR2182075 (2006f:35205)

13. T. Hmidi and S. Keraani: Incompressible viscous flows in borderline Besov spaces, Archive for Rational Mechanics and Analysis, 189, pages 283-300 (2009). MR2413097 (2009j:35252)

14. M. Jellouli: Equation d'Euler sur un domaine extérieur. Existence locale et apparition de singularités, Osaka Journal of Mathematics, 38, pages 619-647 (2001). MR 1860843 (2003b:35175)

15. K. Kikuchi: The existence and uniqueness of nonstationary ideal incompressible flow in exterior domains in $\mathbb{R}^{n}$, Journal of the Mathematical Society of Japan, 38(4), pages 575-598 (1986). MR856127 (87m:35174)

16. X. Liu, M. Wang and Z. Zhang: Local well-posedness and blow-up criterion of the Boussinesq equations in critical Besov spaces, Journal of Mathematical Fluid Mechanics, 12, pages 280292 (2010). MR2645152(2011e:35307) 
17. H. C. Pak and Y. J. Park: Existence of solution for the Euler equations in a critical Besov space $B_{\infty, 1}^{1}\left(\mathbb{R}^{n}\right)$, Communications in Partial Differential Equations, 29, pages 1149-1166 (2004). MR2097579 (2005g:35247)

18. H. Triebel: On Besov-Hardy-Sobolev spaces in domains and regular elliptic boundary value problems. The case $0<p<\infty$, Communications in Partial Differential Equations, 3(12), pages 1083-1164 (1978). MR512083 (81b:46051)

19. M. Vishik: Hydrodynamics in Besov spaces, Archive for Rational Mechanics and Analysis, 145, pages 197-214 (1998). MR 1664597 (2000a:35201)

20. W. Wolibner: Un théorème d'existence du mouvement plan d'un fluide parfait, homogène, incompressible, pendant un temps infiniment long, Mathematische Zeitschrift, 37, pages 698726 (1933). MR.1545430

21. V. Yudovich: Non-stationary flows of an ideal incompressible fluid, Akademija Nauk SSSR. Žurnal Vyčislitel'noŭ Matematiki i Matematičeskoŭ Fiziki, 3, pages 1032-1066 (1963). MR0158189 (28:1415)

22. Y. Zhou: Local well-posedness for the incompressible Euler equations in the critical Besov spaces. Annales de l'Institut Fourier, 54(3), pages 773-786 (2004). MR2097422(2006e:35271)

Université Paris-Est, LAMA, UMR 8050, 61 avenue du GÉnÉral de Gaulle, 94010 Créteil Cedex, France

E-mail address: danchin@univ-paris12.fr 